$\underline{\xi}=-1$

\title{
Eslba Load Sharing Technique for Reputation Manager in Multiple Gateways
}

\author{
${ }^{1}$ T. John Paul Antony, ${ }^{2}$ S. P. Victor \\ ${ }^{*}$ Research Scholar, Bharathiar University, Coimbatore- 641046, Tamilnadu, India \\ ${ }^{2}$, Dean of Science \&amp; Associate Professor in Computer Science, St. Xavier's College, \\ (Autonomous), Palaymkottai, Tamilnadu, India \\ *Corresponding author E-mail: johnpaulinbox@gmail.com
}

\begin{abstract}
Every node has the states of its neighbors such as Selfish, Unselfish and No Information state. The selfish node free path is constructed by avoiding selfish node in the route discovery process. Watch Dog mechanism helps to avoid such issues. Price and Reputation system (P\&RS) helps to diminish the selfish nodes in a successful manner. Trustable node with low portability will go about as a Notoriety Manager and it keeps every one of the records of every node in a record system. The overload of RA leads to various problem and makes the communication very slow. In this paper we discuss about the RA affiliation and request in various possible ways. RA sends the affiliation to the nearest relay node to act as a RA for few nodes. Moreover we discuss the RA communication with Internet through Gateways and the load balancing algorithm - Energy Share Load Balancing Algorithm (ESLBA). The heap adjusting algorithm shares the heap between the nodes and channelizes the ideal course smarterly.
\end{abstract}

Keywords: Reputation Administrator, Load balancing, Relay node, optimal route.

\section{Introduction}

A mobile ad hoc network (MANET) [1] is a remote interconnection of nodes that gives compactness and correspondence between hubs without a central substance like a base station or a passageway point. This empowers the adaptable nodes to setup down to earth frameworks quickly and with no settled topology. At first suggested for use in military applications, MANET can in like manner accept a fundamental part in alleviation(relief) and safeguard operations and moreover in various other individual zone organizing applications like gathering meeting. In the progression of MANET, we have watched that most of the present works have acknowledged a lone remain solitary MANET. Versatile Specially appointed systems are progressively normal and as the application advances the system loads considered for MANETs are likewise expanding [5].A mobile ad hoc network (MANET) is a get-together of remote compact hubs surrounding a short framework without the assistance of any establishment or concentrated association. In spite of the fact that MANETs have advantages and are generally used to shape temporary systems for moment correspondences, they additionally confront a few difficulties because of their specific attributes. The most troublesome test is the constrained energy asset. In the event that a few nodes in the system are abused without legitimate administration, quick energy exhaustion of these nodes will influence appropriate control and nature of administration (QoS) of the entire system.

Wireless sensor networks (WSNs), which are extraordinary class of specially appointed systems [1], are currently utilized broadly for some reasons. A WSN comprises of number of sensor nodes which sense information from the encompassing condition. The gathered information are then sent to the base station which thusly conveys it to the observers [2,3]. WSN is thought to be selfconfigured and foundation less remote system which demonstrates a general WSN with the detecting nodes are conveyed in the location region, the detected information is accumulated by aggregated nodes and sent through remote media to base station or errand administration node which conveys it to the client. At the same time anyone can use the adaptability gave by MANET and the colossal administrations and information gave by the Internet, it would be more alluring. In this way, associating MANET into the Internet is ending up increasingly popular.

When MANET is associated into the Internet, the passages are the section purposes of all activity between the crossover systems such as hybrid networks. Interfacing MANET into the Web is ending up increasingly mainstream. At the point when MANET is associated into the Web, the portals are the section purposes of all activity between the cross breed systems. Typically, a lot of activity load may end up noticeably gathered in some entryways and clog occurs.To decide this issue, this paper proposes a four conceivable methods for the choosing transfer nodes. Through reenactment, we demonstrate that the proposed calculation can convey the activity stack consistently among multiple gateways. As we probably are aware, there are massive complexities among MANET and the Web, so an unprecedented device which goes about as an "expansion" between these two heterogeneous frameworks is required. This remarkable contraption is known as an entryway. Each one of the parcels from/to the Web is coordinated by the passage.

Past works have exhibited that guard dogs are suitable instruments to distinguish acting up and narrow minded nodes. Basically, guard dog frameworks catch remote movement and dissect it to choose whether neighbour nodes are acting in a selfish way [2].

At the point when the watchdog distinguishes a narrow minded node it is set apart as a positive discovery (or a negative location, 
on the off chance that it is recognized as a non-childish node). By the by, watchdogs can flop on this identification, creating false positives and false negatives that truly corrupt the conduct of the system.

\section{Reputation Administrator}

In the below diagram the node RA has to be assumed as a reputation administrator or reputation manager because of the low mobility in the network. The DAT (DISSEMINATED ACCOUNT TABLE) help the notoriety director to gather the notoriety data and exchange data of every portable hub in the MANET [1].

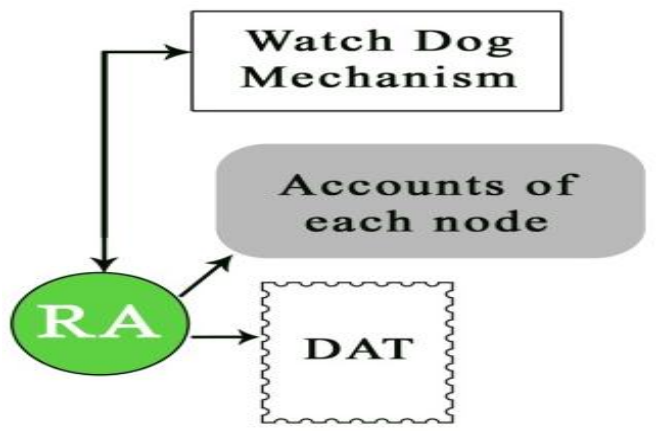

Fig 1: Reputation administrator in P\&RS mechanism

RA holds the details of all nodes in the network, pricing system of all nodes and maintains the DAT with the help of Watch Dog mechanism. Each reputation administrator keeps up the record for each adaptable centre adversary which it is manageable. The reputation regard (Reputation Value) is enlisted by the director and according to the regard (value), the credits in the record of the nodes for those the head is careful will be extended or lessened. The reputation administrator mention that, the nodes that they are having the reputation regard below the constrain and having inadequacy account as uncooperative nodes [1]. The reputation and Pricing system gives a solution to the selfish nodes so a strategically imperative frame can be developed in various forms. Approaching the selfish nodes, entering into to the selfish node circle, selfish node link are the major possibilities in the scenario. For that pricing \& Reputation system can be used in the reputation management.

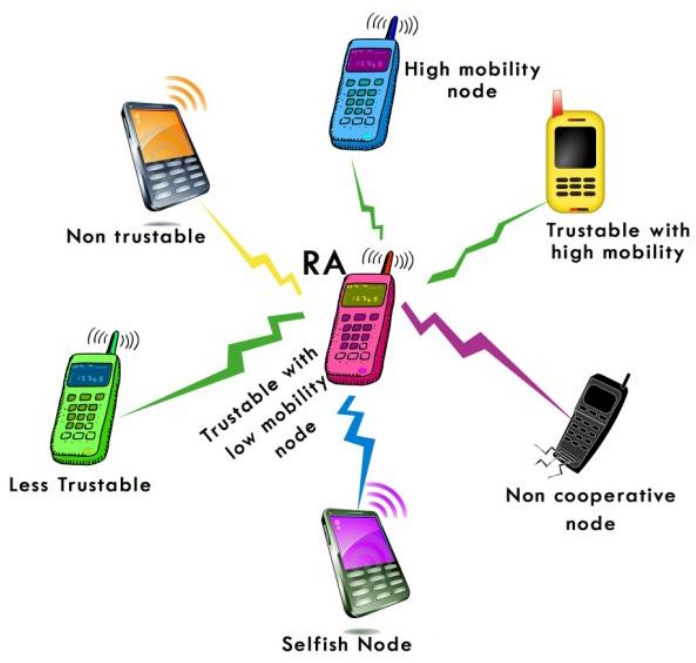

Fig 2: Reputation Administrator

At the point when the P\&RS framework identify that the specific node is uncooperative node, that node is put into the blacklist. The staying portable hubs have mindful of the nodes that they are available operating at a profit list. In the event that any demand is begun from the nodes in the blacklist it suggests it naturally ignore the request [1]

\section{Proposed Work}

To conserve resources or to balance the load among the mobile nodes in the MANET by load balancing algorithm. In past stage, we told that the node with most noteworthy notoriety esteem is chosen as the hand-off node. Thus, there is a circumstance that, a solitary hub with most elevated notoriety esteem is chosen as the transfer node for different circumstances. If a node selected as a relay node for multiple number of times (Greater than threshold), it leads to heavy load for the administrator node so this paper shares the load in various ways by handovering or sharing its operation to other nodes.

The RA sends the affiliation request to its neighbor nodes. The neighbor node will reply to the affiliation request with its reputation value. The node break down the answer message and handover its sending operation to the node with most elevated notoriety esteem by sending the ID of neighbour node with most noteworthy notoriety incentive to the earlier node in the way. In many cases the reputation administrator finds the relay node within the region or within the shortest distance. The high reputation value node will be considered as a relay node and it shares the affiliation to the corresponding node for the further communication. But sometimes the nodes within the region fail to act as a relay node because of the no reputation value. The administrator couldn't find the relay node within the region so RA communicates with the global network for relay node.

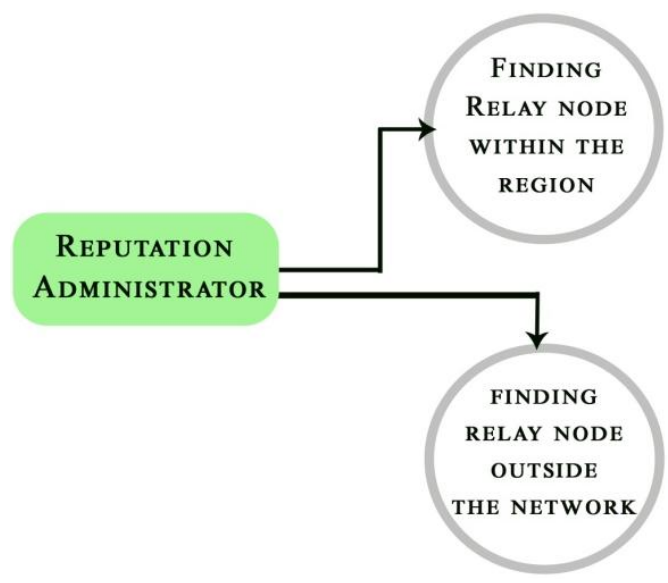

Fig 3: RA architecture

The RA connects with the Internet through a proper gateway to find the relay node with high reputation value. The process will happen in 4 possible ways

Route $1:$ RA $\rightarrow$ Within the region

Route $2:$ RA $\rightarrow$ Outside the region (Internet)

Route 3: RA $\rightarrow$ Gateway $\rightarrow$ Node with high reputation value

Route 4: RA $\rightarrow$ Multiple Gateway $\rightarrow$ Single node or Multiple Node

\subsection{Reputation Administrator within the Region:}

The selfish node can be detected through watch dog mechanism to channelize the work flow of the communication. Reputation administrator acts as a mediator and gathers all the information of the corresponding nodes in the network and stores the information in a separate table called DAT. The account and the pricing system will be maintained by the administrator for the smoother communication and process . The reputation Administrator acts as 
a key role in the network. But sometimes RA fails to act smarter because of the heavy load. So RA shares the affiliation to the neighboring node as a relay node. RA can find the relay node within the network and gives the affiliation request. The high reputation value node will receive the affiliation request and sends the response to act as a relay node.

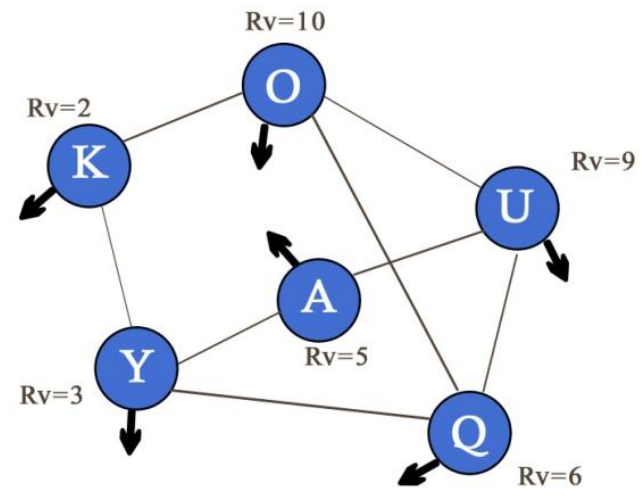

Fig 4: Nodes with reputation values

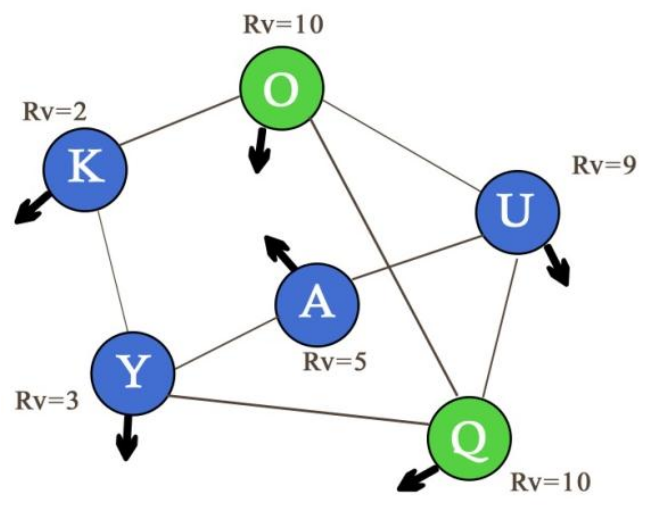

Fig 5: Nodes having same reputation values

It can be defined as

\section{Case 1: RA $\rightarrow\{$ R1: 1 to $\mathbf{n}\} \rightarrow \mathbf{R n}$}

In case of nodes having same reputation values, the RA choose the shortest route node then,

\section{Case 2: $R A \rightarrow\{R 1: 1$ to $n\} \rightarrow R n(O, Q)$}

$\mathbf{R A} \rightarrow\{\mathbf{R} 1: 1$ to $\mathbf{n}\} \rightarrow \mathbf{R n}\{(\operatorname{node}, \mathbf{R v})\} \rightarrow(\operatorname{Rn}\{(\mathbf{O}, 10)(\mathbf{Q}, 10)\}$

i.e., $(\mathbf{O}, 10) \in(Q, 10)$

RA - Relay node, R1 - region one (nearest region), 1to $\mathrm{n}$ - all nodes, $\mathrm{Rn}$ - relay node, Rn (node, RV), RV - Reputation Value.

\subsection{Reputation Administrator with Global Network}

RA communicates the node outside of the region i.e., Internet or Global Network to find the relay with the proper condition. The relay node should have the high reputation value than the other nodes. If the networks have $\mathbf{n}$ number of nodes and $\mathbf{m}$ number of relay nodes then the RA will choose the nearest node as a relay node for the further affiliation. Normally the global network consist of $n$ number of nodes with a different topology. The RA finds the nearest node through shortest path and sends the affiliation. If the nearest node accepts the affiliation and sends the reply then RA will share the permission. Otherwise it will go for next nearest relay node for affiliation request. The below diagram explains the RA communication with the nearest relay node $X_{1}$ with high reputation value. In case of no response with $\mathrm{X}_{1}$ then RA will move on to the next nearest possible node $\mathrm{H}_{1}$ and so on .

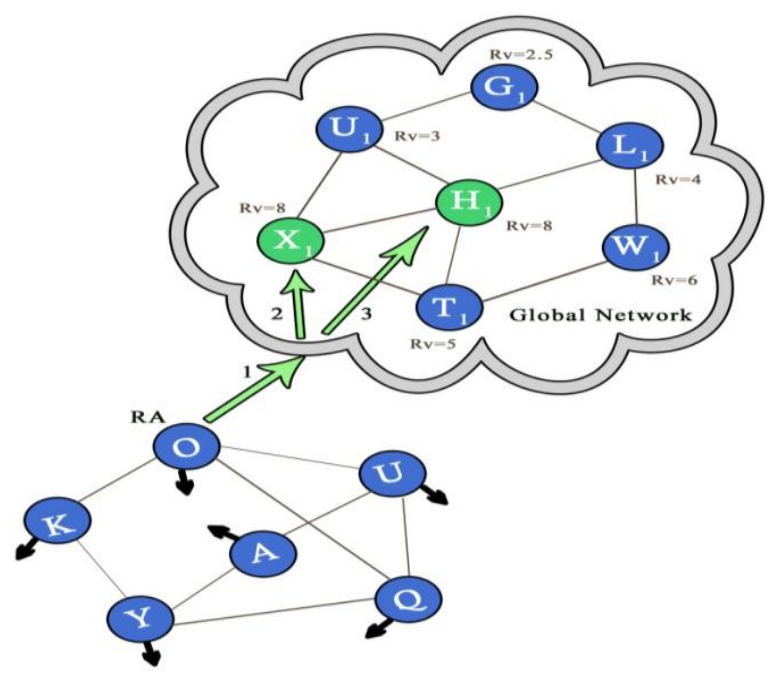

Fig 6: Multiple relay nodes within the Global network

It can be defined as

$\mathrm{RA} \rightarrow\{$ G1: 1 to $\mathbf{n}\} \rightarrow \mathbf{R n}$ (or) $\mathrm{RA} \rightarrow\{$ G1: 1 to $\mathbf{n}\} \rightarrow \operatorname{Rn}($ node 1$)$ $\in \mathbf{R n}\left(X_{1}\right)$

$\mathrm{RA} \rightarrow\{$ G1: 1 to $n\} \rightarrow \operatorname{Rn}$ (or) $\mathrm{RA} \rightarrow\{$ G1: 1 to $n\} \rightarrow \operatorname{Rn}\left(X_{1}, 8\right)$

In case of multiple relay nodes, then

$\mathbf{R A} \rightarrow\{$ G1: 1 to $\mathbf{n}\} \rightarrow \mathbf{R n}$ (or) $\mathbf{R A} \rightarrow\{$ G1: 1 to $\mathbf{n}\} \rightarrow \mathbf{R n}(\mathbf{n} 1, \mathbf{n} 2)$ $\in \mathbf{R n}\left(\mathbf{X}_{1}, \mathbf{H}_{1}\right)$

$\mathbf{R A} \rightarrow\{$ G1: 1 to $\mathbf{n}\} \rightarrow \mathbf{R n} \quad$ (or) $\mathbf{R A} \rightarrow\{$ G1: 1 to $\mathbf{n}\} \rightarrow \mathbf{R n}(\mathbf{n} 1, \mathbf{n} 2)$ $R n\{($ node1,Rv $)($ node2,Rv $)\} \rightarrow R n\left\{\left(X_{1}, 8\right)\left(H_{1}, 8\right)\right\}$ i.e., $\quad\left(X_{1}, 8\right)$ $\in\left(H_{1}, 8\right)$

$\mathrm{n} 1, \mathrm{n} 2$ will be node1, node2 within the Network

RA - Relay node, G1 -Global Network all nodes, $\mathrm{Rn}$ - relay node, RV - Reputation Value.

\subsection{Reputation Administrator with Single Gateway}

Reputation administrator links with the internet through a proper gateway. Versatile nodes need to enlist with one of the passages so as to speak with journalist node. In order to register with a gateway, mobile nodes first need to discover available gateways. Therefore, gateway discovery is a paramount issue in Integrated Internet-MANET. Customary door revelation procedures like proactive, receptive and cross breed [3] should be improved keeping in mind the end goal to adjust to the changing systems administration conditions. Proficient portal revelation components called versatile entryway disclosure instruments have been proposed which play out the errand of door revelation productively.

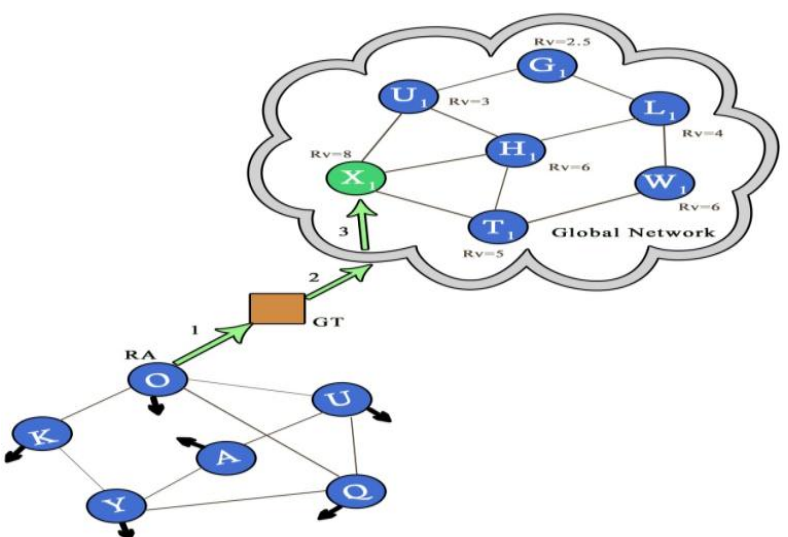

Fig 7: Reputation Administrator link with Internet through gateway 
$\mathrm{RA} \rightarrow \mathrm{Gt} \rightarrow\{$ G1:1to $\mathrm{n}\} \rightarrow \mathrm{Rn}$ (or) $\mathrm{RA} \rightarrow\{$ G1: 1 to $n\}$ $\rightarrow \operatorname{Rn}($ node 1$) \in \operatorname{Rn}\left(X_{1}\right)$

$\mathrm{RA} \rightarrow \mathrm{Gt} \rightarrow\{\mathrm{G1:} 1$ to $\mathrm{n}\} \rightarrow \mathrm{Rn} \quad$ (or) $\mathrm{RA} \rightarrow\{$ G1: 1 to $\mathrm{n}\}$ $\rightarrow \operatorname{Rn}(\mathrm{n} 1, \mathrm{n} 2) \in \mathbf{R n}\left(X_{1}, \mathrm{H}_{1}\right)$

Where Gt means Gateway and here single gateway is mentioned in the above diagram, RA - Relay node, G1 -Global Network (Internet), 1 to $\mathrm{n}$ - all nodes, $\mathrm{Rn}$ - relay node , $\mathrm{Rv}-$ Reputation Value.

\subsection{Reputation Administrator with Multiple Gateways}

RA can be connected with the network in multiple gateways. The RA will do the same process of the single gateway in multiple ways.

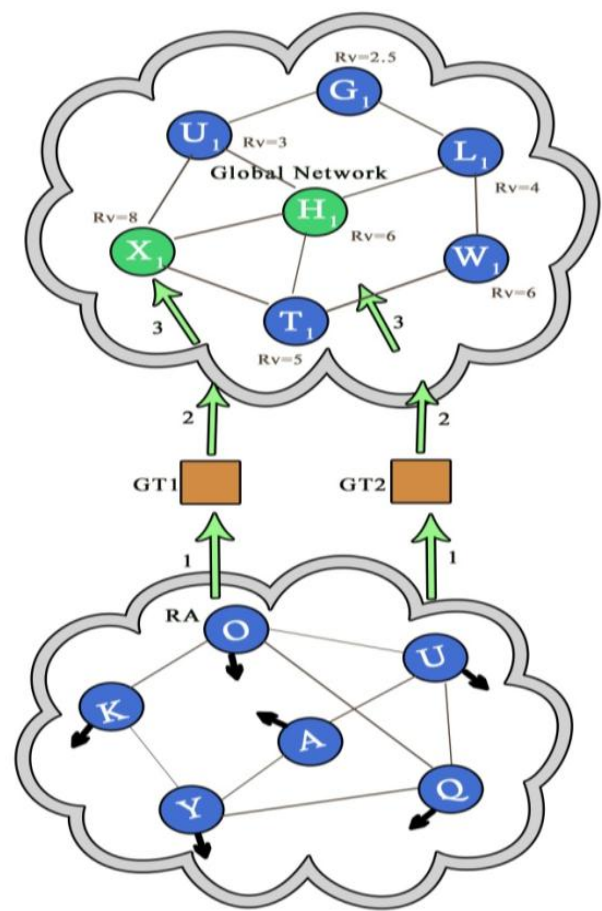

Fig 8: Reputation Administrator link with multiple gateways

Multiple gateway can be used many times for the process of RA $\rightarrow\{\mathrm{G} 1: 1$ to $\mathrm{n}\}$ and it can be mentioned as

$\mathrm{RA} \rightarrow($ Gt1,Gt2) $\rightarrow\{$ G1:1to $\mathrm{n}\}$ Rn (or) $\mathrm{RA} \rightarrow\{$ G1: 1 to $\mathbf{n}\}$ $\rightarrow \operatorname{Rn}(\operatorname{node} 1) \in \operatorname{Rn}\left(X_{1}\right)$

$\mathrm{RA} \rightarrow($ Gt1,Gt2) $\rightarrow\{$ G1: 1 to $\mathrm{n}\} \rightarrow \mathbf{R n} \quad$ (or) $\mathrm{RA} \rightarrow\{$ G1: 1 to $\mathbf{n}\}$ $\rightarrow \operatorname{Rn}(\mathbf{n} 1, \mathrm{n} 2) \in \operatorname{Rn}\left(X_{1}, \mathbf{H}_{1}\right)$

Where Gt means Gateway and here single gateway is mentioned in the above diagram, Gt 1 and Gt2 are the gateways, RA - Relay node,G1 -Global Network

( Internet), 1to $\mathrm{n}$ - all nodes, $\mathrm{Rn}$ - relay node , Rv - Reputation Value.

\section{Load Balancing Technique}

Load changing is method of isolating the work done by the PC between no less than two PCs with the objective that mindboggling work can be administered successfully at a comparative measure of time [6]. Load changing is the most direct strategy for scaling out an application server establishment. If one server is over-trouble then the requesting are sent to another server to process with more prominent breaking point The inspiration driving burden changing is to upgrade the execution of a framework through a legitimate dispersal of the heap to the entire network[7]. A general meaning of this issue is given as takes after: for the given broad number of occupations, we ought to streamline the framework in perspective of load (e.g. mean execution time).

\subsection{Random Load Balancing}

In this methodology the load is balanced by appropriating the load indiscriminately finished the unmistakable servers that may appear in the framework, by picking one discretionary server over the framework and after that the load is balanced [6]. Exactly when this approach is associated with stack altering methodology, the load is balanced effectively.

\subsection{Scheduling Load Balancing}

For stack adjusting calculations, at first we should guarantee their reasonable convenience and furthermore assess them by utilizing reproductions or by utilizing usage over the system [6]. There are two sorts of booking calculation

\subsection{Round-Robin Scheduling}

Round-Robin Scheduling is the synchronous planning calculation. It permits disseminating the load consecutively crosswise over various servers show in arrange. By utilizing this booking calculation, every one of the servers are dealt with as equivalent with no respect to limit or load. This sort of booking calculation takes after the round robin.

\subsection{Weighted Round-Robin Scheduling}

Weighted Round-Robin Planning is the synchronous booking figuring. This count licenses to flow the heap progressively across finished different server appear in orchestrate yet it gives more loads to servers to process with more imperative capacity [8]. The utmost of server is appeared by a customer selected weight factor, which can be adjusted either upward or plummeting by special load task.

\subsection{Evolutionary Load Balancing}

Nature propelled algorithm: This calculation depends on the nature or nature motivated things. In view of the viewpoints of the nature the amusement is played. Ex Mother flying creature encouraging other little birds[9,1 0]. The issue in the amusement is the way the mother feathered creature sustain other winged creature similarly. The mother fledgling can nourish different winged creatures by one by one or haphazardly or flying creatures which request sustenance first. Case for transformative calculation is subterranean insect state improvement, feline swarm advancement, categorize and so on.

\section{Energy Share Load Balancing Algorithm (Eslba)}

Most of the vitality share stack adjusting calculation think about the extra vitality of nodes, and try to restrict the aggregate vitality utilization or lift the framework lifetime. Regardless, most by far of them make an effort not to consider as far as possible and load assignment in the framework. In certified frameworks, a few hubs are as often as possible picked as the sending hubs because of their positions or some different favourable circumstances. Substantial load on the nodes realizes the advantages weariness, for instance, information exchange limit, battery vitality and memory stockpiling. If a node is congested, it can provoke more bundle misfortune, cut down throughput and longer end-to-end delay. Once the vitality of the node runs out, it will cause the course breakage and system parcel. Therefore, stack altering or load adjusting issue should be considered in the directing calculation to 
upgrade the framework execution.

In our Manet model, nodes are arbitrarily appropriated and move unpredictably speeds. Each hub has its intriguing Macintosh address and is outfitted with a lone receiving wire and half-duplex remote handsets. All hubs in the framework eat up settled transmission control when they send the control groups and data bundles. A MANET can be addressed as an undirected graph $(V$, where $V$ is the course of action of hubs and $E$ is the game plan of associations partners the hubs. $\forall n \mid n \in, n$ has a related remaining vitality $\mu$, and size of the movement as Itr. Ibuf is the greatest support estimate and is the same for all nodes [4].

There can be a couple of routes between any two nodes, i.e., node $\mathrm{U}$ and node $\mathrm{G}$. So the way will be $(\mathrm{U}, \mathrm{G})=\alpha 1+\alpha 2+\ldots .+\alpha$ n where each $\alpha i$ is a part way amongst $U$ and $G$. To get an ideal course the way needs to think about vitality ,load and jump tally .Let $\lambda \mathrm{min}$ be the base estimation of $\lambda$ on the way, $\lambda \min =\min \{\lambda i<i<\mathrm{K}\}$ where $\mathrm{K}$ is the arrangement of nodes on the way. Give $\lambda$ to a chance to be the aggregate outstanding vitality of all nodes on the way, which is characterized as $\lambda$ total $=\Sigma n-1 i=0 . \lambda$ th is the limit of the remaining vitality of every hub in the system [4]. Give $\lambda$ a chance to be the base estimation of $\lambda$ on the way, $\lambda \min =\min \{$ $\lambda i \mid i \in N\}$ where $N$ is the arrangement of nodes on the way. Give $\lambda$ total a chance to be the aggregate lingering vitality of all nodes on the way, which is characterized as $\lambda$ total $=\Sigma n-1 i=0 \lambda$. $\lambda$ th is the limit of the leftover vitality of every node in the system.

\section{Description of Eslba}

ESLBA is gotten from specially appointed on request separate vector steering convention [11]. At the point when the correspondence between source node to goal occurs as per the beneath procedure. If a source node sends a message or request to the destination node in the same network or in any other network, it has to check the route whether it is a valid route or not. The source node, RA in this paper sends the request ( RARQ) to any other relay node to some other network then the RA checks the route signal. Many times the intermediate nodes between the source node to destination node receives the RARQ and acts accordingly. But RA never accepts the response(RARP) from the intermediate nodes. First it checks the destination node, then only it allows the next node to respond.

The packet or information needs to monitor the below fields : a) Type of the Route Route ${ }_{\text {type }}, \lambda_{\text {min }}, \lambda_{\text {total }}$

If the intermediate node responds in a channel then the source node has to go through the following process.

1) It will Compare $\lambda$ with $\lambda$ th. If $\lambda<\lambda$ th, Routetype is referred to EL. Something else, Routetype is alluded to $E H$ for all RARQs as what is at first set. Analyze the base leftover vitality field of the RARQ bundle with its own particular remaining vitality and refresh it. On the off chance that $\lambda<\lambda$, supplant the present estimation of $\lambda \min$ with the estimation of $\lambda$ to locate the base leftover vitality on the way.

2) Compare Ktraff with Kbuff. If $\frac{\text { Ktraff }}{\text { Kbuff }} \geq \varepsilon$, the node will communicate the RREQ with a postponement keeping in mind the end goal to keep the conceivable blockage. This postponement is ascertained as $\underline{K \text { traff }} \times T c$, where $T c$ is a Kbuff

constant delay.

3) On the off chance that Routetype is EH, figure the aggregate remaining vitality from the primary node to itself on the present way by adding its own lingering vitality to $\lambda$ total.

\begin{tabular}{|l|l|l|l|l|l|}
\hline \multicolumn{2}{|l|}{ Path Comparison and Route Selection } \\
\hline Path & $\begin{array}{l}\text { Route } \\
\text { Name }\end{array}$ & $\begin{array}{l}\lambda \\
\text { total }\end{array}$ & Delay & $\begin{array}{l}\text { Hop } \\
\text { Count }\end{array}$ & $\begin{array}{l}\text { Weight } \\
\text { Value W }\end{array}$ \\
\hline Green & $\mathrm{S} \rightarrow \mathrm{Y} \rightarrow \mathrm{U}$ & 88 & 0.6 & 4 & 36.6 \\
\hline Red & $\mathrm{S} \rightarrow \mathrm{V} \rightarrow \mathrm{U}$ & 70 & 0.4 & 3 & 58.3 \\
\hline Pink & $\mathrm{S} \rightarrow \mathrm{L} \rightarrow \mathrm{U}$ & 120 & 0.8 & 5 & 30 \\
\hline
\end{tabular}

In order to achieve both sensible activity stack transmission and longer system lifetime, the course assurance technique in view of particular conditions is associated. At the point when the goal hub gets a RARQ packet, it sits tight for a specific measure of time before answering with a RARP parcel so as to get other RARQ parcels. Among all got RARQ bundles, which are moreover the part way between the source and objective center points or nodes, the perfect course is the weight regard. Here, we portray $W$ as the weight estimation of a way. $W$ has unmistakable definitions as the way situation changes. Decision is made on the goal node as takes after:

$$
W=\frac{\lambda \text { total }}{\text { delay } * \text { hop }}
$$

The ideal course is the way with the greatest $W$. On the off chance that more than one way has a similar estimation of $W$, the ideal course is the way with the most brief end-to-end delay.

\section{Performance Analysis in Eslba}

Figure 10 shows the comparison of the routes and load sharing between the nodes. While sharing the loads, the node has to check the delay, hop count and the successive path. The optimal route is the path with maximum $W$.

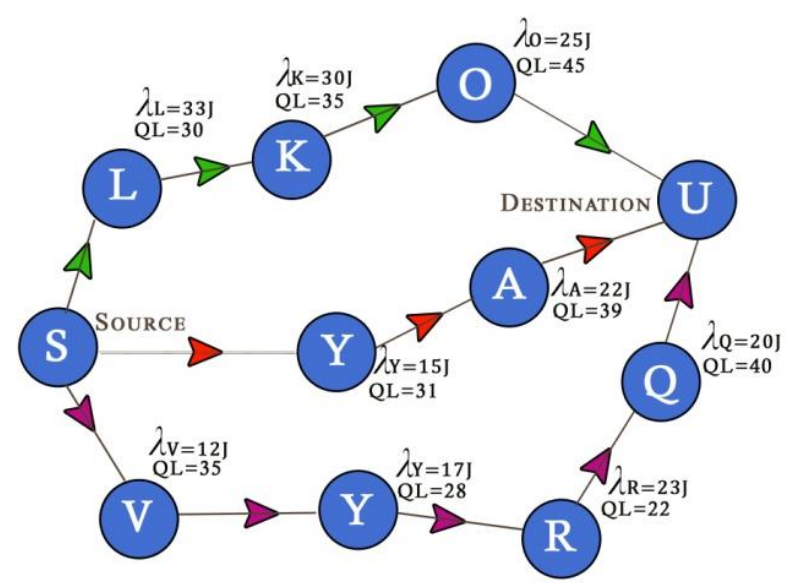

Fig 9: Queue length of each node in different paths

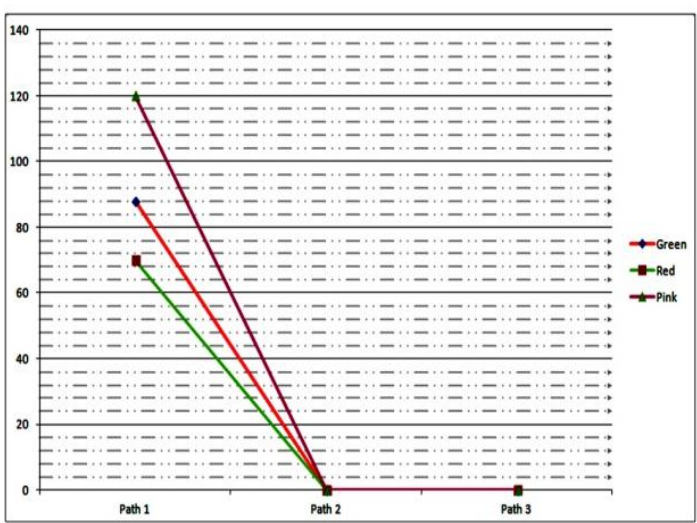

Fig 10: Path Comparison and route selection

The figure 7 explains the ESLBA route sharing between the 
source and destination in the different paths. The destination point $\mathrm{U}$ receives the RARQ from three different paths, i.e., Path 1 $: \mathrm{S} \rightarrow \mathrm{Y} \rightarrow \mathrm{U}(\mathrm{S} \rightarrow \mathrm{L} \rightarrow \mathrm{K} \rightarrow \mathrm{O} \rightarrow \mathrm{U})$, Path $2: \mathrm{S} \rightarrow \mathrm{V} \rightarrow \mathrm{U}(\mathrm{S} \rightarrow \mathrm{Y} \rightarrow \mathrm{A} \rightarrow \mathrm{U})$ , Path $3: S \rightarrow V \rightarrow U(S \rightarrow V \rightarrow Y \rightarrow R \rightarrow Q \rightarrow Q) . \lambda$ is the current energy, QL is the queue length , The threshold energy level $\lambda^{\text {th }}$ is minimum of $10 \mathrm{~J}$ and maximum of $49 \mathrm{~J}$. By comparing all three path weight value the optimal route can be selected. In the above diagram when comparing Green,Red,Pink path, the weight value of red is having bigger weight value. so according to our consideration path red is considered as a optimal route.

Fig 11 explains the $\lambda$ total of three different paths and their nodes. It can be mentioned as $\lambda$ total. The individual joule of each node has been added and mentioned as $\lambda$ total. According to the table values path 3 has the maximum $\lambda$ total, the remaining path has lesser $\lambda$ total when compared to the path3.

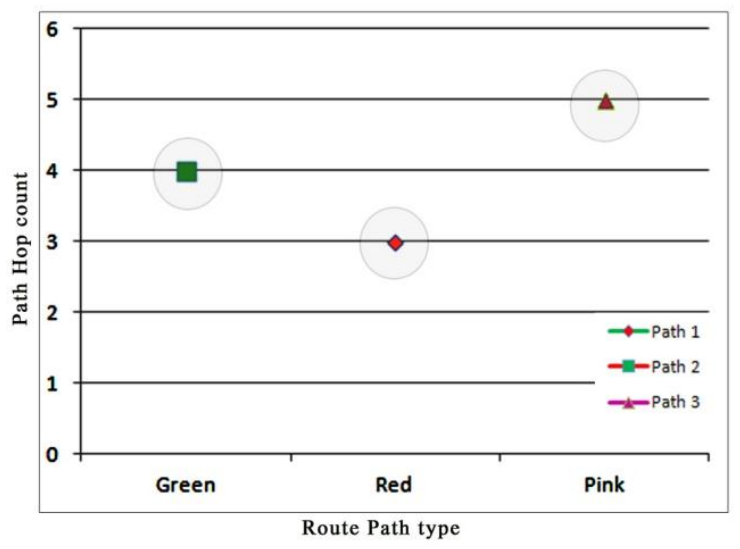

Fig 11: Hop count in the entire network

Fig 11 clearly explains about the hop count of the three path. Here the tree path green, red and pink have nodes from

$\mathrm{S} \rightarrow \mathrm{U}$. The number of nodes between each path can be mentioned as hop count. Path 1 (Green) contains 4 hop counts, Path 2 (Red) contains 3 hop counts and Path 3 ( Pink) contains 5 hop counts.

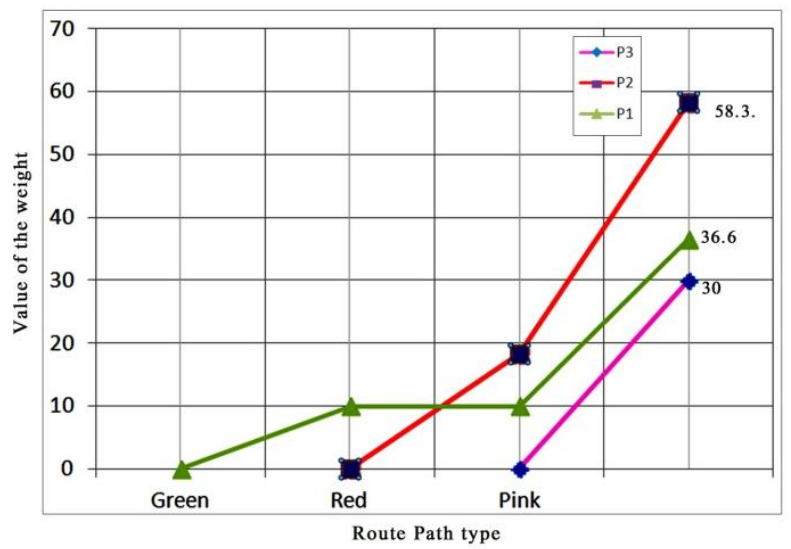

Fig 12: Nodes weight in multiple path

Figure 12 explains the weight of the each node in the path. The weight can be calculated as

$$
W=\frac{\lambda \text { total }}{\text { delay } * \text { hop }}
$$

The green path have the weight value of 36.6, the red path has the weight value of 58.3 and the Path 3 i.e., Pink has the weight value of 30 . According to the weight of the path, the optimal route selection is selected.

\section{Conclusion}

The reputation Administrator channelizes the load through ESLBA algorithmatic technique and balance the load in a optimal route. The affiliation request (RARQ) and the response(RARP) will be happened not only in a single path, it may happen in a multiple ways. The above simulation result and the technique clearly explains the optimal route path selection.

\section{References}

[1] Diminishing the selfish nodes by reputation and pricing system through SRA scenario.

[2] CoCoWa: A Collaborative Contact-based Watchdog for Detecting Selfish Nodes Enrique Hern'andez-Orallo, Manuel D. Serrat, JuanCarlos Cano, Carlos T. Calafate, Pietro Manzoni Departamento de Inform ‘atica de Sistemas y Computadores. Universitat Polit`ecnica de Val’encia. Spain.

[3] Hamidian A., "A Study of Internet Connectivity for Mobile Ad Hoc Networks in NS2", Masters Thesis, Department of Communication Systems, Lund Institute of Technology, Lund University, (2003)

[4] Performance Improvement of Energy- aware MANET Routing Algorithm using Load-Balancing Xiaoying Zhang ,Northeastern University, Shenyang, China, Alagan Anpalagan ,WINCORE Laboratory, Lei Guo School of Information Science and Engineering, Northeastern University, Shenyang, China , 2014 IEEE 17th International Conference on Computational Science and Engineering.

[5] Reduced Energy Consumption And Cooperative Load Balancing Via Reliable Routing In Cluster Based MANETs Jayashree Lakshmi, Arshiya Sultana , IEEE International Conference On Recent Trends In Electronics Information Communication Technology, May 20-21, 2016, India

[6] D. Sudipto, R. Rajesh, D. Pradip, "Optimizations to multipath routing protocols in mobile ad hoc networks", in: Proceedings of the International Conference on Emerging Applications of IT (EAIT 2014), 2014.

[7] Bragard, Q. "Global dynamic load-balancing for decentralised distributed simulation" Published in: Simulation Conference (WSC), 2014

[8] Sandeep Sharma, Sarabjeet Singh, Meenaksshi Sharma, "Performance Analysis of Load Balancing Algorithms", World Academy of Science, Engineering and Technology, 2014.

[9] Ivanoe De Falco , Eryk Laskowski, Richard Olejnik , Umberto Scafuri , Ernesto Tarantino, Marek Tudll.lj Extremal Optimization Applied to Load Balancing in Execution of Distributed Programs" 12 February 2015

[10] N. Mi, Q. Zhang, A. Riska, E. Smirni, "Load balancing for performance diflerentiation in dual-priority clustered servers", in: the 3rd International Conference on the Quantitative Evaluation of Systems (QEST'06), 2006, Riverside, CA, pp. 385-395.

[11] D. B. Johnson and D. A. Maltz, "The dynamic source routing protocol for mobile ad hoc networks", IETF RFC 4728, 1999. 